DOMINIK KMIECIK, JoAnna KobUs-CisowsKa, KLAUdia Kotecka, MoniKa PRZEOR, BARTOSZ KULCZYŃSKI

Department of Food Service and Catering

Poznań University of Life Sciences

\title{
EVALUATION OF THE FAT CONTENT AND FATTY ACID PROFILE INCLUDING TRANS FATTY ACIDS (TFA) IN CONFECTIONERY PRODUCTS AVAILABLE ON THE POLISH MARKET
}

\author{
OCENA ZAWARTOŚCI TŁUSZCZU I PROFILU KWASÓW TŁUSZCZOWYCH, \\ W TYM IZOMERÓW TRANS KWASÓW TŁUSZCZOWYCH (TFA), \\ W WYROBACH CUKIERNICZYCH DOSTEPNYCH NA POLSKIM RYNKU
}

\begin{abstract}
Summary
Background. Confectionery products are easily available and very popular but their nutritional value depends on their composition. Using palm oil and hydrogenated fats in the production process leads to a high content of saturated fatty acids (SFA) and trans fatty acids (TFA) in the product. The aim of the study was to evaluate the fat content and fatty acid profile including trans fatty acids in the popular confectionery products available on the Polish market.

Material and methods. 27 products divided into four groups were subjected to an analysis: chocolate-coated wafers with a filling (nine), wafers with a filling without the chocolate coat (seven), bars (three), as well as breakfast snacks (eight). The product's fat content was defined and the profile of fatty acids was marked using the gas chromatography technique.

Results. The evaluated products were characterized by varied content of fat which oscillated from 8.95 to $31.02 \%$. The participation of the individual fatty acids groups in both wafer groups and bars was close to each other. The composition of the fatty acids in case of breakfast snacks was varied and dependent upon the product type. SFA oscillated from 9.87 to $80.30 \%$, MUFA from 16.24 to $63.67 \%$, PUFA from 3.57 to $29.54 \%$ and TFA from 0.12 to $16.87 \%$.

Conclusions. The evaluated chocolate-covered wafers, those without chocolate and bars contained a higher content of fat and SFA share than the breakfast snacks. Majority of the tested products contained more than $50 \%$ SFA. MUFA were prevailing only in five of the eight evaluated breakfast snacks. Majority of the products were characterized by a low level, less than $2 \%$ of isomers of TFA.
\end{abstract}

Key words: fat content, trans fatty acids (TFA), saturated fatty acids (SFA), confectionery products, wafers, confectionery breakfast snacks 
Kmiecik, D., Kobus-Cisowska, J., Kotecka, K., Przeor, M., Kulczyński, B. (2016). Evaluation of the fat content and fatty acid profile including trans fatty acids (TFA) in confectionery products available on the Polish market. Nauka Przyr. Technol., 10, 4, \#52. DOI: http://dx.doi.org/10.17306/J.NPT.2016.4.52

\section{Introduction}

Presently, confectionery products are very popular. Convenience, size of a serving and price make the products desirable, frequently being a stimulant or creating a need to eat something sweet. Fat content (energetic value) and its type used in the production process (content of SFA and TFA) may pose some objections. Trans isomers of fatty acids in the human diet may come from two sources. The first one are naturally TFA occurring in animal-origin products, mainly milk, butter, and in ruminants' meat. They are produced in small amounts in animals' organisms as a result of natural biohydrogenation processes. The other source are TFA built as a result of the industrial hydrogenation process of oils (industrial-TFA, i-TFA) (Norhayati et al., 2011; Shingfield et al., 2008).

Both groups are perceived differently though, taking into consideration the way they influence the human organism. Natural TFA, including CLA (conjugated linoleic acids), can positively influence the organism via increasing the integrity and efficiency of cells, or staying neutral. Moreover, they do not exhibit any connection with the coronary vessels arteriosclerosis or may perform an antioxidative or anti-cancerous action (Przybojewska and Rafalski, 2003). Consumption of industrial-TFA unfavourably influences the blood lipid profile changes increasing a risk of the cardiovascular diseases and increasing the insulin resistance. They may lead to a weakening of the ability to concentrate, body mass gain, disorders of the prenatal development of babies, nervous system, bring about a constricted reproduction, as well as earlier births (Dorfman et al., 2009; Jamioł-Milc et al., 2010; Larqué et al., 2001). The main source of i-TFA in the diet are products fried on hydrogenated oils, as well as confectionery fats commonly used in the production of pastries, cookies, wafers, sweet fillings and creams. The participation of TFA in these products is significantly varied though, and may oscillate from values close to 0 up to a few tens percent of the total fat value.

The first countries to introduce the compulsory marking of trans isomers content in products were Canada (2003) and the USA (2006). TFA content below $0.2 \mathrm{~g}$ per serving in Canada, and below 0.5 in the USA are permitted to get a TFA-free label (Krettek et al., 2008). In the European Union, legal regulations regarding the content of trans isomers in the products appeared only in a few countries. The first country to introduce the limitations was Denmark (2004), next Switzerland, Austria, Iceland, Norway, Hungary and Sweden, limiting the TFA content at the level of $2 \mathrm{~g}$ per $100 \mathrm{~g}$ of fat or oil (WHO, 2015).

Despite a lack of legal regulations in numerous countries a constant decrease in the TFA content in products has been observed for many years as a result of decreasing participation of TFA in industrial fats used for their production. According to the IMACE European Margarine Association (2016), the number of margarines with the maximum $2 \%$ TFA level increased from 29 to $93 \%$ in the period 2004-2015. The aim of the study was an evaluation of the fat content and fatty acid profile including TFA in the popular confectionery products available on the Polish market. 
Kmiecik, D., Kobus-Cisowska, J., Kotecka, K., Przeor, M., Kulczyński, B. (2016). Evaluation of the fat content and fatty acid profile including trans fatty acids (TFA) in confectionery products available on the Polish market. Nauka Przyr. Technol., 10, 4, \#52. DOI: http://dx.doi.org/10.17306/J.NPT.2016.4.52

\section{Material and methods}

\section{Material}

27 products were subjected to an analysis. They were commonly accessible brand products, as well as brands of supermarkets offered for sale. The products were divided into four groups: chocolate-coated wafers with a filling (nine), wafers with a filling without the chocolate coat (seven), bars (containing wafer and filling) (three), as well as breakfast snacks (eight). Two single packages of each of the product were purchased (30-140 g), coming from two various production parts, and constituted two separate samples. The analysis was carried out between June and December, 2015.

\section{Methods}

\section{Fat content analysis}

The content of fat was marked applying a semi-automatic HT6 1043 Soxtec Extraction Unit (Foss) using the extraction-gravimetric method. A product sample was crushed, placed in an extraction thimble with purified sea sand and extracted using petroleum benzine. The content of fat was calculated from the difference of the sample mass and the quantity of the extracted fat.

\section{Fat extraction from the products}

The extraction of fat was conducted by Folch method (Folch et al., 1957). A sample was comminuted in a mill, next homogenized in the chloroform-methanol mixture $(2: 1, \mathrm{v}: \mathrm{v})$ and separated in a separatory funnel. The chloroform layer was transferred into glass flask and the solvent was evaporated on a vacuum evaporator at $40^{\circ} \mathrm{C}$. The obtained fat was closed under nitrogen and stored at $-24^{\circ} \mathrm{C}$ till the fatty acids profile analysis was carried out.

\section{Fatty acid composition analysis}

The fatty acids profile was marked according to the AOCS official method Ce $1 \mathrm{~h}-05$ (AOCS, 2009b). A fat sample was dissolved in hexane, transesterified with sodium methylate, and next the methyl esters of fatty acids (FAME) were analysed applying the Agilent 7820A GC (Agilent Technologies) gas chromatograph. The chromatograph was equipped with SLB-IL 100 column (Supelco, $100 \mathrm{~m} \times 0.25 \mathrm{~mm} \times 0.2 \mu \mathrm{m}$ ) and FID (flame ionization detector). The carrier gas was helium of $1 \mathrm{ml} / \mathrm{min}$ flow. The temperature of the injector and detector was $250^{\circ} \mathrm{C}$. The sample was separated at the temperature from $120^{\circ} \mathrm{C}$ to $200^{\circ} \mathrm{C}$ in the split mode $(1: 10)$ in 90 min time. The fatty acids (FA) were identified by comparison of their retention times with commercially available standards.

\section{Calculation of the iodine value}

The calculated iodine value was defined directly from the fatty acids composition according to the AOCS Cd 1c-85 method (AOCS, 2009a). 
Kmiecik, D., Kobus-Cisowska, J., Kotecka, K., Przeor, M., Kulczyński, B. (2016). Evaluation of the fat content and fatty acid profile including trans fatty acids (TFA) in confectionery products available on the Polish market. Nauka Przyr. Technol., 10, 4, \#52. DOI: http://dx.doi.org/10.17306/J.NPT.2016.4.52

\section{Statistical analysis}

All assays were performed in two replications. Values of means and standard deviations were calculated with the use of Microsoft Office Excel 2013 (Microsoft Corporation). The significance of differences between means was determined at $p<0.05$ using the analysis of variance (ANOVA) followed by Tukey's multiple range test with STATISTICA PL 10.0 (StatSoft, Inc.).

\section{Results and discussion}

The fat content in the analysed products was varied and oscillated from 8.95 to $31.02 \%$. The slight differences were found between the chocolate-covered and non-covered wafers. The fat content in the first group oscillated from 24.96 to $31.02 \%$ (Table 1) and in the second group from 22.60 to $30.49 \%$ (Table 2). The fat content in bars

Table 1 . The fatty acid profile, fat content and iodine value of the analysed chocolate coated wafers with a filling

Tabela 1. Profil kwasów tłuszczowych, zawartość tłuszczu oraz liczba jodowa analizowanych wafelków z nadzieniem oblanych czekoladą

\begin{tabular}{|c|c|c|c|c|c|c|}
\hline $\begin{array}{l}\text { No. } \\
\text { Lp. }\end{array}$ & $\begin{array}{c}\Sigma \text { SFA } \\
(\%)\end{array}$ & $\begin{array}{c}\Sigma \text { MUFA } \\
(\%)\end{array}$ & $\begin{array}{c}\Sigma \text { PUFA } \\
(\%)\end{array}$ & $\begin{array}{c}\Sigma \mathrm{TFA} \\
(\%)\end{array}$ & $\begin{array}{c}\text { Fat content } \\
\text { Zawartość } \\
\text { tłuszczu } \\
(\%)\end{array}$ & $\begin{array}{c}\text { Iodine value } \\
\text { Liczba jodowa }\end{array}$ \\
\hline 1 & 2 & 3 & 4 & 5 & 6 & 7 \\
\hline $1 \mathrm{~A}$ & $59.28 \pm 1.57 \mathrm{ab}$ & $33.94 \pm 0.68 \mathrm{ab}$ & $6.78 \pm 0.38 \mathrm{acd}$ & $0.32 \pm 0.02 \mathrm{a}$ & $28.44 \pm 0.71 \mathrm{ad}$ & $41.15 \pm 0.65 \mathrm{abd}$ \\
\hline 1B & $60.83 \pm 0.56 \mathrm{bd}$ & $32.63 \pm 0.13 \mathrm{ab}$ & $6.53 \pm 0.26 \mathrm{acd}$ & $0.40 \pm 0.04 \mathrm{a}$ & $27.35 \pm 0.42 \mathrm{ae}$ & $39.62 \pm 0.48 \mathrm{abd}$ \\
\hline $2 \mathrm{~A}$ & $56.95 \pm 0.30 \mathrm{ad}$ & $35.99 \pm 0.33 \mathrm{abd}$ & $7.06 \pm 0.05 \mathrm{~cd}$ & $1.32 \pm 0.07 \mathrm{a}$ & $28.36 \pm 0.24 \mathrm{ad}$ & $43.51 \pm 0.37 \mathrm{bd}$ \\
\hline $2 \mathrm{~B}$ & $57.97 \pm 1.39 \mathrm{ab}$ & $35.19 \pm 2.46 \mathrm{abc}$ & $6.84 \pm 0.36 \mathrm{acd}$ & $1.69 \pm 0.05 \mathrm{ab}$ & $27.92 \pm 0.35 \mathrm{a}$ & $42.44 \pm 0.61 \mathrm{abd}$ \\
\hline $3 \mathrm{~A}$ & $57.41 \pm 1.23 \mathrm{ad}$ & $37.64 \pm 0.74 b d$ & $4.96 \pm 0.29 \mathrm{cbcd}$ & $6.72 \pm 0.05 \mathrm{ab}$ & $28.02 \pm 0.07 \mathrm{ad}$ & $41.34 \pm 1.15 \mathrm{abd}$ \\
\hline 3B & $58.69 \pm 1.03 \mathrm{ab}$ & $36.33 \pm 1.52 \mathrm{abd}$ & $4.98 \pm 0.04 \mathrm{abcd}$ & $5.34 \pm 0.04 \mathrm{ab}$ & $28.14 \pm 0.02 \mathrm{ad}$ & $39.96 \pm 1.22 \mathrm{abd}$ \\
\hline $4 \mathrm{~A}$ & $63.00 \pm 1.72 b$ & $31.26 \pm 0.40 \mathrm{a}$ & $5.74 \pm 0.39 \mathrm{acd}$ & $0.36 \pm 0.03 \mathrm{a}$ & $28.73 \pm 1.41 \mathrm{ab}$ & $37.17 \pm 0.32 \mathrm{a}$ \\
\hline $4 \mathrm{~B}$ & $63.28 \pm 0.79 b$ & $30.75 \pm 0.78 \mathrm{a}$ & $5.97 \pm 0.17 \mathrm{acd}$ & $0.28 \pm 0.02 \mathrm{a}$ & $28.70 \pm 0.35 \mathrm{ab}$ & $37.11 \pm 0.86 \mathrm{a}$ \\
\hline $5 \mathrm{~A}$ & $58.20 \pm 2.07 \mathrm{ad}$ & $35.38 \pm 0.58 \mathrm{abc}$ & $6.42 \pm 0.26 \mathrm{acd}$ & $0.36 \pm 0.02 \mathrm{a}$ & $27.30 \pm 0.34 \mathrm{ae}$ & $41.79 \pm 1.10 \mathrm{abd}$ \\
\hline $5 \mathrm{~B}$ & $57.51 \pm 1.22 \mathrm{ad}$ & $35.16 \pm 0.09 \mathrm{abc}$ & $7.34 \pm 0.24 \mathrm{ad}$ & $0.49 \pm 0.05 a$ & $28.26 \pm 0.40 \mathrm{ae}$ & $43.24 \pm 0.18 \mathrm{bd}$ \\
\hline $6 \mathrm{~A}$ & $54.94 \pm 0.36 \mathrm{a}$ & $41.37 \pm 1.78 \mathrm{~d}$ & $3.69 \pm 0.06 b$ & $7.61 \pm 0.06 b$ & $30.93 \pm 0.57 b$ & $40.39 \pm 2.90 \mathrm{abd}$ \\
\hline $6 \mathrm{~B}$ & $54.93 \pm 1.82 \mathrm{a}$ & $40.46 \pm 0.59 \mathrm{~cd}$ & $4.61 \pm 0.36 \mathrm{abc}$ & $7.80 \pm 0.04 b$ & $31.02 \pm 0.70 b$ & $42.88 \pm 1.34 \mathrm{abd}$ \\
\hline 7A & $33.44 \pm 0.96 \mathrm{c}$ & $60.98 \pm 1.56 \mathrm{e}$ & $5.58 \pm 0.06 \mathrm{abcd}$ & $16.84 \pm 0.04 \mathrm{c}$ & $24.96 \pm 1.09 \mathrm{c}$ & $63.31 \pm 0.51 \mathrm{c}$ \\
\hline 7B & $35.42 \pm 0.93 c$ & $60.16 \pm 3.83 \mathrm{e}$ & $4.42 \pm 0.04 \mathrm{abc}$ & $16.84 \pm 0.08 \mathrm{c}$ & $25.42 \pm 0.61 \mathrm{ce}$ & $59.70 \pm 1.08 \mathrm{c}$ \\
\hline $8 \mathrm{~A}$ & $60.58 \pm 0.68 b$ & $35.37 \pm 1.90 \mathrm{abc}$ & $4.05 \pm 0.01 \mathrm{c}$ & $2.28 \pm 0.05 \mathrm{ab}$ & $28.96 \pm 0.16 \mathrm{ab}$ & $37.69 \pm 0.31 \mathrm{a}$ \\
\hline
\end{tabular}


Kmiecik, D., Kobus-Cisowska, J., Kotecka, K., Przeor, M., Kulczyński, B. (2016). Evaluation of the fat content and fatty acid profile including trans fatty acids (TFA) in confectionery products available on the Polish market. Nauka Przyr. Technol., 10, 4, \#52. DOI: http://dx.doi.org/10.17306/J.NPT.2016.4.52

Table 1 - cont. / Tabela $1-$ cd.

\begin{tabular}{|c|l|l|l|l|l|l|}
\hline 1 & \multicolumn{1}{|c|}{2} & 3 & 4 & 5 & 6 & \multicolumn{1}{c|}{7} \\
\hline 8B & $57.71 \pm 1.73 \mathrm{ad}$ & $37.25 \pm 0.56 \mathrm{bd}$ & $5.04 \pm 0.05 \mathrm{abcd}$ & $3.02 \pm 0.06 \mathrm{ab}$ & $30.35 \pm 0.71 \mathrm{bd}$ & $40.93 \pm 1.35 \mathrm{abd}$ \\
$9 \mathrm{~A}$ & $56.26 \pm 2.12 \mathrm{a}$ & $36.04 \pm 0.67 \mathrm{abd}$ & $7.70 \pm 0.04 \mathrm{~d}$ & $0.22 \pm 0.01 \mathrm{a}$ & $30.08 \pm 0.02 \mathrm{~b}$ & $44.64 \pm 0.72 \mathrm{~d}$ \\
9B & $61.92 \pm 1.22 \mathrm{bd}$ & $31.63 \pm 0.36 \mathrm{ab}$ & $6.46 \pm 0.09 \mathrm{ad}$ & $0.54 \pm 0.01 \mathrm{a}$ & $31.02 \pm 0.28 \mathrm{~b}$ & $38.79 \pm 0.68 \mathrm{ab}$ \\
\hline
\end{tabular}

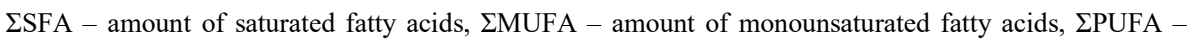
amount of polyunsaturated fatty acids, $\Sigma$ TFA - amount of trans isomers of fatty acids.

Means in the same column followed by different letters indicate significant statistical differences $(\mathrm{p} \leq 0.05)$.

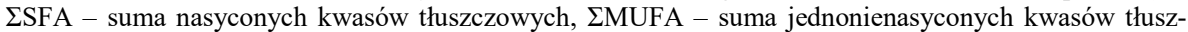

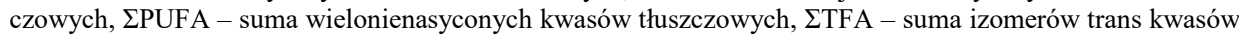
thuszczowych.

Średnie w tej samej kolumnie oznaczone różnymi literami wskazują na istotne różnice statystyczne $(\mathrm{p} \leq 0,05)$.

Table 2. The fatty acid profile, fat content and iodine value of the analysed wafers with a filling without chocolate

Tabela 2. Profil kwasów tłuszczowych, zawartość tłuszczu oraz liczba jodowa analizowanych wafelków z nadzieniem bez czekolady

\begin{tabular}{|c|c|c|c|c|c|c|}
\hline $\begin{array}{l}\text { No. } \\
\text { Lp. }\end{array}$ & $\begin{array}{c}\Sigma \mathrm{SFA} \\
(\%)\end{array}$ & $\begin{array}{c}\Sigma \text { MUFA } \\
(\%)\end{array}$ & $\begin{array}{c}\Sigma \text { PUFA } \\
(\%)\end{array}$ & $\begin{array}{c}\Sigma \mathrm{TFA} \\
(\%)\end{array}$ & $\begin{array}{c}\text { Fat content } \\
\text { Zawartość } \\
\text { thuszczu } \\
(\%)\end{array}$ & $\begin{array}{l}\text { Iodine value } \\
\text { Liczba jodowa }\end{array}$ \\
\hline $1 \mathrm{~A}$ & $66.93 \pm 1.02 \mathrm{a}$ & $28.45 \pm 0.52 \mathrm{a}$ & $4.61 \pm 0.08 \mathrm{a}$ & $0.16 \pm 0.01 \mathrm{a}$ & $30.30 \pm 0.28 \mathrm{a}$ & $32.62 \pm 0.23 a$ \\
\hline $1 \mathrm{~B}$ & $65.36 \pm 0.92 \mathrm{a}$ & $29.79 \pm 0.71 \mathrm{a}$ & $4.85 \pm 0.04 \mathrm{a}$ & $0.16 \pm 0.02 \mathrm{a}$ & $30.17 \pm 0.83 \mathrm{ae}$ & $34.19 \pm 0.61 \mathrm{a}$ \\
\hline $2 \mathrm{~A}$ & $74.63 \pm 0.18 b$ & $21.56 \pm 0.25 b$ & $3.82 \pm 0.14 b$ & $0.04 \pm 0.00 \mathrm{a}$ & $29.16 \pm 0.61 \mathrm{ade}$ & $25.20 \pm 0.35 b$ \\
\hline $2 B$ & $75.54 \pm 1.27 b$ & $20.82 \pm 0.52 b$ & $3.64 \pm 0.09 b$ & $0.09 \pm 0.00 \mathrm{a}$ & $29.33 \pm 0.17 \mathrm{ade}$ & $24.31 \pm 0.51 b$ \\
\hline $3 \mathrm{~A}$ & $53.61 \pm 1.85 \mathrm{c}$ & $38.08 \pm 0.79 c$ & $8.30 \pm 0.36 c$ & $4.16 \pm 0.10 b$ & $25.64 \pm 0.21 b$ & $47.45 \pm 1.26 \mathrm{c}$ \\
\hline $3 B$ & $53.61 \pm 1.07 \mathrm{c}$ & $38.04 \pm 0.53 \mathrm{c}$ & $8.36 \pm 0.30 c$ & $3.78 \pm 0.01 \mathrm{c}$ & $25.24 \pm 0.35 b$ & $47.43 \pm 0.72 c$ \\
\hline $4 \mathrm{~A}$ & $50.26 \pm 0.18 \mathrm{c}$ & $39.77 \pm 0.06 \mathrm{c}$ & $9.97 \pm 0.10 \mathrm{de}$ & $0.40 \pm 0.03 \mathrm{a}$ & $22.60 \pm 0.13 \mathrm{c}$ & $51.61 \pm 0.10 \mathrm{de}$ \\
\hline $4 \mathrm{~B}$ & $51.63 \pm 0.25 c$ & $38.61 \pm 0.27 \mathrm{c}$ & $9.77 \pm 0.13 \mathrm{de}$ & $0.37 \pm 0.02 \mathrm{a}$ & $23.02 \pm 0.04 \mathrm{cf}$ & $50.25 \pm 0.05 \mathrm{~d}$ \\
\hline $5 \mathrm{~A}$ & $52.28 \pm 1.67 \mathrm{c}$ & $38.15 \pm 1.24 \mathrm{c}$ & $9.57 \pm 0.41 \mathrm{~d}$ & $0.18 \pm 0.01 \mathrm{a}$ & $28.01 \pm 0.20 \mathrm{~d}$ & $49.52 \pm 1.10 \mathrm{~cd}$ \\
\hline $5 \mathrm{~B}$ & $51.29 \pm 0.91 c$ & $39.04 \pm 0.12 \mathrm{c}$ & $9.67 \pm 0.08 \mathrm{~d}$ & $0.27 \pm 0.02 \mathrm{a}$ & $28.20 \pm 0.77 \mathrm{de}$ & $50.40 \pm 0.08 \mathrm{~d}$ \\
\hline $6 \mathrm{~A}$ & $50.28 \pm 0.31 \mathrm{c}$ & $39.79 \pm 0.17 \mathrm{c}$ & $9.93 \pm 0.03 \mathrm{de}$ & $0.41 \pm 0.02 \mathrm{a}$ & $25.04 \pm 1.02 \mathrm{bf}$ & $51.56 \pm 0.02 \mathrm{de}$ \\
\hline $6 \mathrm{~B}$ & $48.40 \pm 0.80 \mathrm{c}$ & $41.27 \pm 0.59 c$ & $10.33 \pm 0.21 \mathrm{e}$ & $0.42 \pm 0.03 \mathrm{a}$ & $25.25 \pm 0.71 b$ & $51.96 \pm 2.10 \mathrm{e}$ \\
\hline $7 \mathrm{~A}$ & $65.31 \pm 2.35 \mathrm{a}$ & $28.57 \pm 0.49 \mathrm{a}$ & $6.12 \pm 0.16 f$ & $0.15 \pm 0.01 \mathrm{a}$ & $30.14 \pm 0.27 \mathrm{ae}$ & $32.38 \pm 0.30 \mathrm{a}$ \\
\hline $7 \mathrm{~B}$ & $63.32 \pm 1.48 \mathrm{a}$ & $29.68 \pm 0.82 \mathrm{a}$ & $7.00 \pm 0.17 \mathrm{~g}$ & $0.22 \pm 0.01 \mathrm{a}$ & $30.49 \pm 0.52 \mathrm{a}$ & $37.66 \pm 0.99 f$ \\
\hline
\end{tabular}

Notation - as in Table 1.

Oznaczenia - jak w tabeli 1 . 
Kmiecik, D., Kobus-Cisowska, J., Kotecka, K., Przeor, M., Kulczyński, B. (2016). Evaluation of the fat content and fatty acid profile including trans fatty acids (TFA) in confectionery products available on the Polish market. Nauka Przyr. Technol., 10, 4, \#52. DOI: http://dx.doi.org/10.17306/J.NPT.2016.4.52

was significantly different and oscillated from 16.81 to 30.19 (Table 3 ). The most varied products group was the breakfast snack, where the fat content was from 8.95 to $26.92 \%$ (Table 4). The dominant group of fatty acids in the analysed chocolate-covered, as well as non-covered wafers and bars were saturated fatty acids (SFA), followed by monounsaturated fatty acids (MUFA) and polyunsaturated fatty acids (PUFA). The percentage participation of the saturated fatty acids in the fat separated from the products was from 33.44 to $63.28 \%$ in the chocolate-covered wafers (Table 1), from 48.80 to $75.54 \%$ in the non-covered ones (Table 2), and from 46.50 to $80.30 \%$ in bars (Table 3). MUFA dominated in one case (product 7) in chocolate-covered wafers group. Only a part of the breakfast snacks products was characterized by a prevalence of SFA (products 4,5 and 7). SFA participation in the breakfast products was from 9.87 to $74.78 \%$ (Table 4), while that of MUFA, depending upon the analysed group, oscillated from 30.75 to $60.98 \%$ in wafers with chocolate, from 20.82 to $41.27 \%$ in filled wafers, from 16.24 to $43.78 \%$ in bars and from 18.58 to $63.67 \%$ in breakfast snacks. PUFA constituted the least numerous group of fatty acids. Their participation in the chocolate-covered wafers oscillated from 3.69 to $7.70 \%$, in the non-covered ones from 3.64 to $10.33 \%$, in bars from 3.57 to $10.01 \%$, and in breakfast snacks from 5.16 to $29.54 \%$ of all the fatty acids. Their lowest participation was observed in the products where SFA participation was higher than $60 \%$. The highest PUFA participation was observed in products in which SFA decreased below $15 \%$. The highest differentiation was observed while evaluating the participation of TFA isomers in the analysed products. Their level in the chocolate-covered wafers oscillated from 0.18 to $16.84 \%$, in non-chocolate wafers from 0.04 to $4.16 \%$, in bars from 0.12 to $0.51 \%$ and in breakfast snacks from 0.14 to $16.74 \%$. The analysis of fatty acids profile of fat separated from the products, indicates an insignificant differentiation of the evaluated wafers, no matter whether chocolate-covered or not, which may indicate that the strongest influence on the wafers fat fraction have the com-

Table 3. The fatty acid profile, fat content and iodine value of the analysed bars

Tabela 3. Profil kwasów tłuszczowych, zawartość tłuszczu oraz liczba jodowa analizowanych batonów

\begin{tabular}{|c|c|c|c|c|c|c|}
\hline $\begin{array}{c}\text { No. } \\
\text { Lp. }\end{array}$ & $\begin{array}{c}\Sigma \text { SFA } \\
(\%)\end{array}$ & $\begin{array}{c}\sum \text { MUFA } \\
(\%)\end{array}$ & $\begin{array}{c}\sum \text { PUFA } \\
(\%)\end{array}$ & $\begin{array}{c}\Sigma \text { TFA } \\
(\%)\end{array}$ & $\begin{array}{c}\text { Fat content } \\
\text { Zawartość } \\
\text { thuszczu } \\
(\%)\end{array}$ & $\begin{array}{c}\text { Iodine value } \\
\text { Liczba jodowa }\end{array}$ \\
\hline 1A & $46.52 \pm 0.16 \mathrm{a}$ & $43.48 \pm 0.08 \mathrm{a}$ & $10.01 \pm 0.05 \mathrm{a}$ & $0.33 \pm 0.05 \mathrm{a}$ & $29.32 \pm 0.35 \mathrm{a}$ & $55.15 \pm 0.13 \mathrm{a}$ \\
$1 \mathrm{~B}$ & $47.04 \pm 1.17 \mathrm{a}$ & $43.74 \pm 0.75 \mathrm{a}$ & $9.24 \pm 0.14 \mathrm{a}$ & $0.38 \pm 0.01 \mathrm{a}$ & $30.19 \pm 0.94 \mathrm{a}$ & $54.18 \pm 1.33 \mathrm{a}$ \\
2A & $77.81 \pm 2.06 \mathrm{~b}$ & $17.48 \pm 0.28 \mathrm{~b}$ & $4.72 \pm 0.15 \mathrm{~b}$ & $0.51 \pm 0.05 \mathrm{a}$ & $19.75 \pm 0.53 \mathrm{~b}$ & $23.74 \pm 1.78 \mathrm{~b}$ \\
2B & $79.37 \pm 2.93 \mathrm{~b}$ & $17.09 \pm 0.96 \mathrm{~b}$ & $3.65 \pm 0.31 \mathrm{~b}$ & $0.40 \pm 0.01 \mathrm{a}$ & $20.42 \pm 0.61 \mathrm{~b}$ & $21.13 \pm 1.61 \mathrm{~b}$ \\
3A & $79.59 \pm 1.45 \mathrm{~b}$ & $16.91 \pm 0.14 \mathrm{~b}$ & $3.60 \pm 0.23 \mathrm{~b}$ & $0.13 \pm 0.02 \mathrm{a}$ & $16.81 \pm 0.01 \mathrm{c}$ & $20.85 \pm 0.28 \mathrm{~b}$ \\
3B & $80.30 \pm 0.48 \mathrm{~b}$ & $16.24 \pm 0.09 \mathrm{~b}$ & $3.57 \pm 0.01 \mathrm{~b}$ & $0.12 \pm 0.00 \mathrm{a}$ & $17.00 \pm 0.07 \mathrm{c}$ & $20.20 \pm 0.09 \mathrm{~b}$ \\
\hline
\end{tabular}

Notation - as in Table 1.

Oznaczenia - jak w tabeli 1 . 
Kmiecik, D., Kobus-Cisowska, J., Kotecka, K., Przeor, M., Kulczyński, B. (2016). Evaluation of the fat content and fatty acid profile including trans fatty acids (TFA) in confectionery products available on the Polish market. Nauka Przyr. Technol., 10, 4, \#52. DOI: http://dx.doi.org/10.17306/J.NPT.2016.4.52

Table 4. The fatty acid profile, fat content and iodine value of the analysed breakfast snacks Tabela 4. Profil kwasów tłuszczowych, zawartość tłuszczu oraz liczba jodowa analizowanych przekąsek śniadaniowych

\begin{tabular}{|c|c|c|c|c|c|c|}
\hline $\begin{array}{l}\text { No. } \\
\text { Lp. }\end{array}$ & $\begin{array}{c}\Sigma \text { SFA } \\
(\%)\end{array}$ & $\begin{array}{c}\Sigma \text { MUFA } \\
(\%)\end{array}$ & $\begin{array}{c}\Sigma P U F A \\
(\%)\end{array}$ & $\begin{array}{c}\Sigma \mathrm{TFA} \\
(\%)\end{array}$ & $\begin{array}{c}\text { Fat content } \\
\text { Zawartość } \\
\text { thuszczu } \\
(\%)\end{array}$ & $\begin{array}{c}\text { Iodine value } \\
\text { Liczba jodowa }\end{array}$ \\
\hline $1 \mathrm{~A}$ & $31.12 \pm 2.31 \mathrm{a}$ & $47.73 \pm 1.02 \mathrm{a}$ & $21.15 \pm 1.11 \mathrm{a}$ & $16.74 \pm 1.02 \mathrm{a}$ & $15.53 \pm 0.12 \mathrm{a}$ & $81.98 \pm 0.86 a b$ \\
\hline 1B & $32.43 \pm 0.10 \mathrm{a}$ & $46.62 \pm 0.13 \mathrm{ai}$ & $20.95 \pm 0.38 \mathrm{a}$ & $16.63 \pm 0.27 \mathrm{a}$ & $14.61 \pm 0.52 \mathrm{ab}$ & $80.62 \pm 0.07 a$ \\
\hline $2 \mathrm{~A}$ & $18.69 \pm 0.15 b$ & $63.52 \pm 0.46 b$ & $17.79 \pm 0.15 b$ & $0.16 \pm 0.15 b$ & $11.98 \pm 0.31 \mathrm{bcd}$ & $84.91 \pm 0.16 b$ \\
\hline $2 \mathrm{~B}$ & $18.46 \pm 1.38 b$ & $63.67 \pm 1.27 b$ & $17.87 \pm 0.21 b$ & $0.18 \pm 0.13 b$ & $11.98 \pm 0.61 \mathrm{abd}$ & $93.30 \pm 1.04 b$ \\
\hline $3 \mathrm{~A}$ & $13.55 \pm 0.51 \mathrm{c}$ & $57.62 \pm 0.32 \mathrm{c}$ & $28.83 \pm 0.32 \mathrm{c}$ & $9.24 \pm 0.17 \mathrm{c}$ & $9.38 \pm 0.23 \mathrm{~cd}$ & $107.80 \pm 0.17 \mathrm{~cd}$ \\
\hline $3 B$ & $14.24 \pm 0.71 \mathrm{c}$ & $56.86 \pm 0.60 \mathrm{c}$ & $28.90 \pm 0.49 c$ & $9.53 \pm 0.11 c$ & $8.95 \pm 0.13 c$ & $107.43 \pm 0.76 \mathrm{~cd}$ \\
\hline $4 \mathrm{~A}$ & $68.36 \pm 2.20 \mathrm{~d}$ & $24.08 \pm 0.63 \mathrm{~d}$ & $7.57 \pm 0.15 d$ & $0.13 \pm 0.04 b$ & $21.49 \pm 1.38 \mathrm{~d}$ & $33.87 \pm 0.25 \mathrm{e}$ \\
\hline $4 B$ & $68.57 \pm 0.70 \mathrm{~d}$ & $23.84 \pm 0.22 \mathrm{~d}$ & $7.59 \pm 0.18 \mathrm{~d}$ & $0.14 \pm 0.08 b$ & $22.35 \pm 0.71 d$ & $33.69 \pm 0.32 \mathrm{e}$ \\
\hline $5 \mathrm{~A}$ & $74.78 \pm 5.25 \mathrm{e}$ & $18.75 \pm 1.28 \mathrm{e}$ & $6.47 \pm 0.61 \mathrm{e}$ & $0.14 \pm 0.06 b$ & $13.58 \pm 0.60 \mathrm{ab}$ & $27.43 \pm 2.13 f$ \\
\hline $5 B$ & $74.69 \pm 2.89 \mathrm{e}$ & $18.58 \pm 1.24 \mathrm{e}$ & $6.73 \pm 0.61 \mathrm{de}$ & $0.25 \pm 0.11 b$ & $13.74 \pm 0.22 \mathrm{ab}$ & $27.84 \pm 1.98 f$ \\
\hline $6 \mathrm{~A}$ & $10.32 \pm 0.68 \mathrm{f}$ & $60.76 \pm 0.43 f$ & $28.92 \pm 0.35 \mathrm{c}$ & $0.57 \pm 0.02 b d$ & $13.17 \pm 0.12 \mathrm{ab}$ & $110.07 \pm 0.31 \mathrm{~cd}$ \\
\hline $6 \mathrm{~B}$ & $9.85 \pm 0.18 \mathrm{f}$ & $60.61 \pm 0.27 f$ & $29.54 \pm 0.84 \mathrm{c}$ & $0.64 \pm 0.74 \mathrm{bd}$ & $13.43 \pm 0.82 \mathrm{ab}$ & $111.01 \pm 0.64 d$ \\
\hline $7 \mathrm{~A}$ & $60.54 \pm 0.54 \mathrm{~g}$ & $33.22 \pm 0.21 \mathrm{~g}$ & $6.24 \pm 0.46 \mathrm{e}$ & $0.82 \pm 0.39 \mathrm{~d}$ & $26.73 \pm 2.23 \mathrm{e}$ & $39.91 \pm 0.48 \mathrm{~g}$ \\
\hline $7 \mathrm{~B}$ & $61.11 \pm 1.33 \mathrm{~g}$ & $33.73 \pm 0.27 \mathrm{~g}$ & $5.16 \pm 0.09 f$ & $0.80 \pm 0.10 \mathrm{bd}$ & $26.92 \pm 0.10 \mathrm{e}$ & $38.07 \pm 0.25 \mathrm{~g}$ \\
\hline $8 \mathrm{~A}$ & $41.34 \pm 0.11 \mathrm{~h}$ & $43.60 \pm 0.15 \mathrm{~h}$ & $15.06 \pm 0.13 \mathrm{~g}$ & $1.73 \pm 0.09 \mathrm{e}$ & $13.33 \pm 0.23 \mathrm{ab}$ & $65.34 \pm 0.03 \mathrm{~h}$ \\
\hline $8 \mathrm{~B}$ & $41.55 \pm 0.83 \mathrm{~h}$ & $44.51 \pm 0.72 \mathrm{hi}$ & $13.94 \pm 0.28 \mathrm{~h}$ & $1.42 \pm 0.02 \mathrm{bd}$ & $13.42 \pm 0.61 \mathrm{ab}$ & $57.56 \pm 0.10 \mathrm{~h}$ \\
\hline
\end{tabular}

Notation - as in Table 1.

Oznaczenia - jak w tabeli 1.

ponents used in the production of their filling mass. The recipe composition also influences the high differences observed in the snacks. The products belonging to the group of the so-called milk sandwiches contained, in majority of cases, from two to a few times more SFA than those composed of confectionery with dried tropical fruits and filling layers. A high content of SFA in confectionery products and bars has been observed for many years in numerous countries all over the world. This is connected with the using of both palm oil and hydrogenated fats for their production (Çakmak et al., 2010; Norhayati et al., 2011; Świderski et al., 2006). The calculated iodine value (CIV) of the fat extracted from the analysed products oscillated from 20.20 (bars) to 111.01 (breakfast snacks). Low CIV's were characteristic for the products rich in SFA. Increase of CIV was related to an increase in oleic and linoleic acids in the composition of the products which may prove that plant oils and non-hydrogenated plant oils were used during their production. Besides the high SFA content, confectionery products are 
Kmiecik, D., Kobus-Cisowska, J., Kotecka, K., Przeor, M., Kulczyński, B. (2016). Evaluation of the fat content and fatty acid profile including trans fatty acids (TFA) in confectionery products available on the Polish market. Nauka Przyr. Technol., 10, 4, \#52. DOI: http://dx.doi.org/10.17306/J.NPT.2016.4.52

a significant source of TFA isomers and of fat in our diet. However, the participation of TFA in a product is dependent upon the type of fat used in its production.

Using hydrogenated fats, being the main source of TFA, in the production process leads to a high level of the TFA in the product. That is why the confectionery products have been viewed, for a long time, as one of the main sources of TFA in the human diet (Kmiecik et al., 2016; Onacik-Gür et al., 2014; Siti Nurshahbani and Azrina, 2014).

However, the situation has been constantly improving. A relatively low level of TFA in many of the evaluated products is a result of the use of fats not subjected to the hydrogenation process but transesterification or recipe modifications (e.g. using shea fat). The tendency has been observed for a few years despite the fact there are no legal regulations concerning the content of TFA in a majority of the world countries (Ansorena et al., 2013; IMACE..., 2016; Richter et al., 2009; Roe et al., 2013; Wagner et al., 2008; WHO, 2015).

\section{Conclusions}

1. The evaluated products contained from 8.95 to $31.02 \%$ of fat. Chocolate-covered wafers, non-covered wafers and bars showed a higher content of fat than the breakfast snacks.

2. The participation of SFA, in a majority of the evaluated products, was more than $50 \%$. Only in the group of breakfast snacks MUFA were prevailing in five of the eight evaluated products.

3. The participation of TFA was varied within each of the analysed groups and oscillated from 0.13 to $16.84 \%$ of the total fat content. $2 \%$ TFA value per $100 \mathrm{~g}$ of fat was exceeded only in seven of the 27 evaluated products.

\section{References}

Ansorena, D., Echarte, A., Ollé, R., Astiasarán, I. (2013). 2012: No trans fatty acids in Spanish bakery products. Food Chem., 138, 1, 422-429. doi: 10.1016/j.foodchem.2012.10.096

AOCS. (2009a). AOCS official method Cd 1c-85. Calculated iodine value. In: V. C. Mehlenbacher, E. M. Sallee, T. H. Hopper, W. E. Link, R. O. Walker (eds.), Official methods and recommended practices of the AOCS. USA: American Oil Chemists' Society.

AOCS. (2009b). AOCS official method Ce 1h-05. Determination of cis-, trans-, saturated, monounsaturated and polyunsaturated fatty acids in vegetable or non-ruminant animal oils and fats by capillary GLC. In: V. C. Mehlenbacher, E. M. Sallee, T. H. Hopper, W. E. Link, R. O. Walker (eds.), Official methods and recommended practices of the AOCS. USA: American Oil Chemists' Society.

Çakmak, Y. S., Güler, G. Ö., Aktümsek, A. (2010). Trans fatty acid contents in chocolates and chocolate wafers in Turkey. Czech J. Food Sci., 28, 3, 177-184.

Dorfman, S. E., Laurent, D., Gounarides, J. S., Li, X., Mullarkey, T. L., Rocheford, E. C., Sari-Sarraf, F., Hirsch, E. A., Hughes, T. E., Commerford, S. R. (2009). Metabolic implications of dietary trans-fatty acids. Obesity, 17, 6, 1200-1207. doi: 10.1038/oby.2008.662 
Kmiecik, D., Kobus-Cisowska, J., Kotecka, K., Przeor, M., Kulczyński, B. (2016). Evaluation of the fat content and fatty acid profile including trans fatty acids (TFA) in confectionery products available on the Polish market. Nauka Przyr. Technol., 10, 4, \#52. DOI: http://dx.doi.org/10.17306/J.NPT.2016.4.52

Folch, J., Lees, M., Sloane Stanley, G. H. (1957). A simple method for the isolation and purification of total lipides from animal tissues. J. Biol. Chem. 226, 1, 497-509. doi: 10.1016/j.ul trasmedbio.2011.03.005

IMACE European Margarine Association. (2016). Margarines have never been lower in trans fatty acids than they are today. IMACE Newsl., 16, 4, 4. http://imace.org/wp-content/uploads/ 2016/10/IMACE_Newsletter_A4_OCT_2016_v41.pdf

Jamioł-Milc, D., Stachowska, E., Chlubek, D. (2010). Skutki spożywania trans nienasyconych kwasów thuszczowych w okresie ciąży i laktacji. Ann. Acad. Med. Stetin. / Rocz. Pom. AM Szczec., 56, 1, 21-27.

Kmiecik, D., Kobus-Cisowska, J., Hęś, M., Szymandera-Buszka, K., Przeor, M. (2016). Smażone produkty ziemniaczane w diecie Polaków jako źródło substancji niekorzystnych żywieniowo. In: K. Melski, D. Walkowiak-Tomczak (eds.), Żywność dla świadomego konsumenta (pp. 112-123). Poznań: Wyd. UP.

Krettek, A., Thorpenberg, S., Bondjers, G. (2008). Trans fatty acids and health: a review of health hazards and existing legislation. Policy Department Economic and Scientific Policy. European Parliament Study. Brussels: Milieu.

Larqué, E., Zamora, S., Gil, A. (2001). Dietary trans fatty acids in early life: a review. Early Hum. Dev., 65, Suppl., 2, S31-S41.

Norhayati, M., Azrina, A., Norhaizan, M. E., Muhammad Rizal, R. (2011). Trans fatty acids content of biscuits commercially available in Malaysian market and comparison with other countries. Int. Food Res. J., 18, 3, 1097-1103.

Onacik-Gür, S., Żbikowska, A., Kowalska, M. (2014). Źródła izomerów trans kwasów thuszczowych na polskim rynku. Probl. Hig. Epidemiol., 95, 1, 120-124.

Przybojewska, B., Rafalski, H. (2003). Kwasy tłuszczowe występujące w mleku a zdrowie człowieka (cz. 4). Kwas wakcenowy cis i trans. Przegl. Mlecz., 9, 343-346.

Richter, E. K., Shawish, K. A., Scheeder, M. R. L., Colombani, P. C. (2009). Trans fatty acid content of selected Swiss foods: The TransSwissPilot study. J. Food Compos. Anal., 22, 5, 479-484. doi: 10.1016/j.jfca.2009.01.007

Roe, M., Pinchen, H., Church, S., Elahi, S., Walker, M., Farron-Wilson, M., Buttriss, J., Finglas, P. (2013). Trans fatty acids in a range of UK processed foods. Food Chem., 140, 3, 427-431. doi: 10.1016/j.foodchem.2012.08.067

Shingfield, K. J., Chilliard, Y., Toivonen, V., Kairenius, P., Givens, D. I. (2008). Trans fatty acids and bioactive lipids in ruminant milk. Adv. Exp. Med. Biol., 606, 3-65. doi: 10.1007/978-0387-74087-4 1

Siti Nurshahbani, S., Azrina, A. (2014). Trans fatty acids in selected bakery products and its potential dietary exposure. Int. Food Res. J., 21, 6, 2175-2181.

Świderski, F., Waszkiewicz-Robak, B., Obiedziński, M., Matias, D. (2006). Jakość rynkowych wyrobów cukierniczych z dużym udziałem tłuszczu. Żywn. Nauka Technol. Jakość, 46, Supl., 1, 192-200.

Wagner, K.-H., Plasser, E., Proell, Ch., Kanzler, S. (2008). Comprehensive studies on the trans fatty acid content of Austrian foods: convenience products, fast food and fats. Food Chem., 108, 3, 1054-1060. doi: 10.1016/j.foodchem.2007.11.038

WHO. (2015). Eliminating trans fats in Europe. A policy brief. Copenhagen: WHO Regional Office for Europe. 
Kmiecik, D., Kobus-Cisowska, J., Kotecka, K., Przeor, M., Kulczyński, B. (2016). Evaluation of the fat content and fatty acid profile including trans fatty acids (TFA) in confectionery products available on the Polish market. Nauka Przyr. Technol., 10, 4, \#52. DOI: http://dx.doi.org/10.17306/J.NPT.2016.4.52

\title{
OCENA ZAWARTOŚCI TŁUSZCZU I PROFILU KWASÓW TŁUSZCZOWYCH, W TYM IZOMERÓW TRANS KWASÓW TŁUSZCZOWYCH (TFA), W WYROBACH CUKIERNICZYCH DOSTĘPNYCH NA POLSKIM RYNKU
}

\begin{abstract}
Streszczenie
Wstęp. Wyroby cukiernicze są łatwo dostępne i bardzo popularne wśród konsumentów, ich wartość odżywcza zależy jednak od składu recepturowego. Wykorzystanie do ich produkcji tłuszczu palmowego oraz tłuszczów uwodornionych może powodować, że są one bogatym źródłem nasyconych kwasów thuszczowych (SFA) oraz izomerów trans kwasów tłuszczowych (TFA). Celem pracy była ocena zawartości tłuszczu oraz profilu kwasów tłuszczowych z uwzględnieniem izomerów trans kwasów tłuszczowych w popularnych produktach cukierniczych dostępnych na polskim rynku.

Material i metody. Analizie poddano 27 produktów podzielonych na cztery grupy: wafelki $\mathrm{z}$ nadzieniem oblane czekoladą (dziewięć), wafelki z nadzieniem bez czekolady (siedem), batony (trzy) oraz przekąski śniadaniowe (osiem). W produkcie określono zawartość tłuszczu oraz oznaczono profil kwasów tłuszczowych, wykorzystując technikę chromatografii gazowej.

Wyniki. Zawartość tłuszczu w ocenianych produktach była zróżnicowana i wynosiła od 8,95 do $31,02 \%$. Udział poszczególnych grup kwasów tłuszczowych w obu grupach wafelków oraz w batonach był zbliżony. W grupie przekąsek śniadaniowych skład kwasów był zróżnicowany i zależał od typu produktu. SFA stanowiły od 9,87 do $80,30 \%$, MUFA - od 16,24 do 63,67\%, PUFA - od 3,57 do $29,54 \%$ i TFA - od 0,12 do $16,87 \%$.

Wnioski. Oceniane wafelki w czekoladzie, bez czekolady i batony charakteryzowały się większą zawartością thuszczu i SFA niż przekąski śniadaniowe. W większości ocenianych produktów udział SFA wynosił ponad 50\%, jedynie w grupie przekąsek śniadaniowych w pięciu na osiem ocenianych produktów przeważały MUFA. Większość produktów charakteryzowała się niskim, poniżej $2 \%$, poziomem izomerów trans kwasów tłuszczowych.
\end{abstract}

Słowa kluczowe: zawartość tłuszczu, kwasy thuszczowe trans (TFA), nasycone kwasy thuszczowe (SFA), wyroby cukiernicze, wafelki, cukiernicze przekąski śniadaniowe

Corresponding address - Adres do korespondencji:

Dominik Kmiecik, Katedra Technologii Żywienia Człowieka, Uniwersytet Przyrodniczy w Poznaniu, ul.Wojska Polskiego 31/33, 60-624 Poznań, Poland, e-mail:dominik.kmiecik@up.poznan.pl

Accepted for publication - Zaakceptowano do opublikowania:

12.12.2016

For citation - Do cytowania:

Kmiecik, D., Kobus-Cisowska, J., Kotecka, K., Przeor, M., Kulczyński, B. (2016). Evaluation of the fat content and fatty acid profile including trans fatty acids (TFA) in confectionery products available on the Polish market. Nauka Przyr. Technol., 10, 4, \#52. DOI: http://dx.doi.org/10.17306/J.NPT. 2016.4.52 